\title{
EURISOL Design Study: Towards an Ultimate ISOL Facility for Europe
}

\author{
$\underline{\text { Y. Blumenfeld }}^{1}$, P. A. Butler ${ }^{2}$, J. C. Cornell 3 , G. Fortuna ${ }^{4}$, M. Lindroos ${ }^{5}$ \\ on behalf of the EURISOL Design Study \\ 1 IPNO, Université Paris Sud, IN2P3-CNRS, 91406 Orsay Cedex, France \\ 2 Oliver Lodge Laboratory, University of Liverpool, Liverpool L69 7ZE, UK \\ ${ }^{3}$ GANIL (CEA/DSM-CNRS/IN2P3) BP 5027, 14076 Caen Cedex 5, France \\ ${ }^{4}$ INFN, Laboratori Nazionale di Legnaro, Legnaro, Italy \\ 5 CERN, AB Department, $\mathrm{CH}-1211$ Genève 22 \\ Contacte-mail:eurisol@ipno.in2p3.fr
}

The scientific promises of radioactive beam research have led the European nuclear physics community to propose to study and build a next generation ISOL facility called EURISOL. A preliminary design study was conducted in the European Union $5^{\text {th }}$ framework programme. The baseline design is a multi-MW proton beam impinging on a liquid mercury converter preceding a fissile target. Spallation targets are also planned. Post-acceleration will be performed with a superconducting heavy-ion LINAC which is designed for 150 $\mathrm{MeV} /$ nucleon ${ }^{132} \mathrm{Sn}$ for example.

More detailed engineering studies and prototype building are currently being carried out in the framework of the EURISOL Design Study[1], supported within the European Union $6^{\text {th }}$ framework programme. Twenty European laboratories and institutions from twelve countries participate in this endeavour. The work is divided into 12 tasks, covering accelerators (driver and post accelerator), high power targets, safety, beam manipulation, beam intensity calculations and physics and instrumentation. A conceptual study for a neutrino beta-beam facility making use of the beta-decay of the large quantities of radioactive nuclei produced is an integral part of the programme.

This talk will describe the baseline design of the EURISOL facility and its expected performances. Some details about recent advances concerning in particular the driver capabilities, the post-accelerator design and the production targets will be given. The place of EURISOL in the roadmap for nuclear physics facilities in Europe in the next two decades will be discussed, in particular in relation to the "intermediate-generation" ISOL facilities - MAFF, HIE-ISOLDE, SPES, and SPIRAL2 - currently planned or under construction.

[1] see http://www.eurisol.org

We acknowledge the financial support of the European Community under the FP6 "Research Infrastructure Action-Structuring the European Research Area" EURISOL DS Project contract no 515768 RIDS. The EC is not liable for the use that can be made of the information contained herein. 